\title{
Targeting eosinophils: another strategy for asthma treatment
}

Hye Mi Jee, MD, PhD

Department of Pediatrics, CHA Bundang Medical Center, CHA University School of Medicine, Seongnam, Korea

\begin{abstract}
Key message
- Identifying phenotypes and endotypes of asthma patients is challenging, and eosinophilic phenotypes are generally characterized by severe or refractory asthma.

Biologicals targeting eosinophils are promising for the control of severe or refractory asthma symptoms.

To ensure proper treatment, increased understanding of the diverse phenotypes of high Th2 inflammation in pediatric asthma is needed.
\end{abstract}

The prevalence of asthma in the 6-to 12-year-old population is $11 \%-13 \%{ }^{1)}$ The majority of asthmatic children have relatively little difficulty controlling their symptoms according to standard guidelines for asthma treatment. However, some children with severe asthma require a second option for controlling asthma symptoms such as high-dose inhaled steroids or systemic corticosteroids. Unfortunately, these children might continue to have poor disease control despite the additional treatment. The prevalence of severe asthma in children is reportedly less than $5 \%$ in the asthmatic population. ${ }^{2)}$

Asthma is currently recognized as a heterogenous disease. Various studies have attempted to define clinical phenotypes and molecular endotypes in asthma patients. ${ }^{1)}$ Most adult patients with severe asthma are categorized as having an eosinophilic phenotype. This is characterized by airway infiltration with eosinophilic inflammatory cells, poor asthma control, frequent exacerbations, and reduced lung function. ${ }^{2)}$ On the other hand, there are currently no universally accepted phenotypes or endotypes in children. Although there are some previous studies on biologic phenotypes in children, categorizing by phenotype is more difficult in children than in adults. ${ }^{3)}$ Interestingly, children with mild to moderate rather than severe asthma had submucosal eosinophilia. ${ }^{4)}$ Bossley et al. ${ }^{3)}$ reported no significant difference in the inhaled or maintenance steroid doses between children with eosinophilic inflammation in the airway and those who did not undergo a bronchoalveolar lavage fluid examination. It is likely that those results differed from those of adults because it was difficult to perform bronchoscopy or sputum induction tests and collect samples in children versus adults. In addition, children with wheezing or asthmatic symptoms often also have a viral infection, which might lead to a neutrophil-dominant status. ${ }^{3)}$ Choi recently reviewed the pathogenesis and treatment of eosinophilic asthma in children. ${ }^{5)}$

Eosinophils, the predominant cell type in severe asthmatic inflammation in the airways that secrete allergic inflammatory cytokines such as interleukin 2 (IL-2), IL-4, IL-5, and IL-10, evolve to Th 2 cytokines. Some studies analyzed pediatric asthma populations and reported 4 or 5 clusters by phenotype ${ }^{6}$ and reported that atopic condition or high allergic sensitization was the dominant phenotype in childhood-onset asthma. ${ }^{7}$

Considering that asthma is a disease with diverse endotypes and phenotypes, personalized treatment by phenotype or endotype has become an important concept of asthma management. Most asthmatic children should start treatment according to welldefined guidelines. If the patient's condition is not controlled after the use of higher dose inhaled corticosteroids or additional treatment, we can consider biological treatment to control the "severe asthma" or "uncontrolled asthma" phenotype. Biological therapy should be considered in patients with this phenotype. To date, 3 biologic therapies (anti-immunoglobulin E, anti-IL-3, and anti-IL-5) have been approved by the U.S. Food and Drug Administration for severe or treatment-refractory asthma. ${ }^{8}$ Anti-IL-5 therapy, such as mepolizumab, reslizumab, and benralizumab, is currently available as eosinophil-targeting agents. Mepolizumab and benralizumab are approved for patients aged 12 years or older, while reslizumab is approved for those aged 18 years or older. ${ }^{5)}$

To evaluate the indications for biological therapies for severe asthma, it is necessary to use a phenotype-based approach and carefully classify comorbidities. If the phenotype shows eosinophilic inflammation, mepolizumab may be a treatment option. If the phenotype is neutrophilic or non-Th2-type inflammation, these treatments are not appropriate. However, Th2 markers and eosinophil levels may not be correlated, and this relationship may change even in the same child. In real practice, we must be aware of this possibility before prescribing biologic

Corresponding author: Hye Mi Jee, MD. PhD. Department of Pediatrics, CHA Bundang Medical Center, CHA University School of Medicine, 59 Yatap-ro, Bundang-gu, Seongnam 13496, Korea 
therapies. ${ }^{9)}$ In terms of monitoring eosinophilic inflammation after anti-IL- 5 treatment, several biomarkers such as peripheral total eosinophil count, fraction of exhaled nitric oxide, and serum eosinophil-derived neurotoxin (EDN) have been suggested. Jang et al. ${ }^{10)}$ reported that EDN level might be a potential biomarker after reslizumab treatment in adult patients with severe eosinophilic asthma.

Although most of the experimental results are limited to adult populations, treatment with anti-IL-5 has brought us into a new era for severe asthma management. Efforts to identify pediatric asthma phenotypes and endotypes and further understand the pathophysiology of childhood asthma must be made to ensure proper treatment.

\section{Conflicts of interest}

No potential conflict of interest relevant to this article was reported.

See the article "Eosinophils and childhood asthma" via https:// doi.org/10.3345/cep.2020.00717.

\section{References}

1. Engelkes M, Baan EJ, de Ridder MAJ, Svensson E, Prieto-Alhambra D, Lapi F, et al. Incidence, risk factors and re-exacerbation rate of severe asthma exacerbations in a multinational, multidatabase pediatric cohort study. Pediatr Allergy Immunol 2020;31:496-505.
2. Chung KF, Wenzel SE, Brozek JL, Bush A, Castro M, Sterk PJ, et al. International ERS/ATS guidelines on definition, evaluation and treatment of severe asthma. Eur Respir J 2014;43:343-73.

3. Bossley CJ, Fleming L, Gupta A, Regamey N, Frith J, Oates T, et al. Pediatric severe asthma is characterized by eosinophilia and remodeling without T(H)2 cytokines. J Allergy Clin Immunol 2012;129:974-82.e13.

4. Payne DN, Qiu Y, Zhu J, Peachey L, Scallan M, Bush A, et al. Airway inflammation in children with difficult asthma: relationships with airflow limitation and persistent symptoms. Thorax 2004;59:862-9.

5. Choi BS. Eosinophils and childhood asthma. Clin Exp Pediatr 2021;64: 60-7.

6. Howrylak JA, Fuhlbrigge AL, Strunk RC, Zeiger RS, Weiss ST, Raby $\mathrm{BA}$, et al. Classification of childhood asthma phenotypes and long-term clinical responses to inhaled anti-inflammatory medications. J Allergy Clin Immunol 2014;133:1289-300.e12.

7. Fitzpatrick AM, Moore WC. Severe asthma phenotypes - how should they guide evaluation and treatment? J Allergy Clin Immunol Pract 2017; 5:901-8.

8. Nelson RK, Bush A, Stokes J, Nair P, Akuthota P. Eosinophilic asthma. J Allergy Clin Immunol Pract 2020;8:465-73.

9. Fleming L, Tsartsali L, Wilson N, Regamey N, Bush A. Longitudinal relationship between sputum eosinophils and exhaled nitric oxide in children with asthma. Am J Respir Crit Care Med 2013;188:400-2.

10. Jang JH, Woo SD, Lee Y, Kim CK, Shin YS, Ye YM, et al. Changes in type 2 biomarkers after anti-IL5 treatment in patients with severe eosinophilic asthma. Allergy Asthma Immunol Res 2021;13:330-8.

How to cite this article: Jee HM. Targeting eosinophils: another strategy for asthma treatment. Clin Exp Pediatr 2021; 64:223-4. https://doi.org/10.3345/cep.2021.00017 